Recibido: junio de 2017

Aprobado: agosto de 2017

DOI: https://doi.org/10.15332/rev.m.v|4i0.2171

Plaza principal de la ciudad de San Juan de Pasto, Nariño, Colombia

Fuente: Carlos Humberto Gómez Arciniegas, 2016.

* Artículo de reflexión

* Profesor asociado de Rehabilitación Estructural de Patrimonio Histórico Construido, Departamento de Arquitectura, DiDA - Universidad de los Estudios de Florencia, Italia; miembro de: Icomos-Cuba; IcoFort/lcomos; Iscarsah/lcomos. Lleva 40 años investigando sobre el comportamiento estructural de arcos y bóvedas de mampostería, y además en técnicas sustentables de consolidación de monumentos históricos. Correo electrónico: michele.paradiso@unifi.it

*** Arquitecto de la Universidad Santo Tomás Bucaramanga, Colombia; especialista en Planificación del Territorio para el Desarrollo. Politécnico di Milano (Italia) - Université de Paris I, Pantheon-Sorbonne, París, Francia; MSc en Relaciones Internacionales y Estudios Estratégicos con énfasis en Políticas de Desarrollo. Lancaster University (Inglaterra), y PhD en Planificación Urbana, Territorial y Ambiental - Politécnico di Milano, Italia. Docente investigador, líder de la línea de investigación en Planificación y Gestión del Territorio, adscrita al grupo de Investigaciones de la Facultad de Arquitectura de la Universidad Santo Tomás Bucaramanga, Colombia. Correo electrónico: carlos.gomez@ustabuca.edu.co

\section{EL DILEMA ENTRE CONSERVACIÓN Y RENOVACIÓN PARA EL DESARROLLO URBANO DE LOS CENTROS HISTÓRICOS. UNA APROXIMACIÓN A SAN JUAN DE PASTO, COLOMBIA*}

\author{
Michele Paradiso** - Università Degli Studi di Firenze, Italia \\ Carlos Humberto Gómez Arciniegas *** - Universidad Santo Tomás, Colombia
}

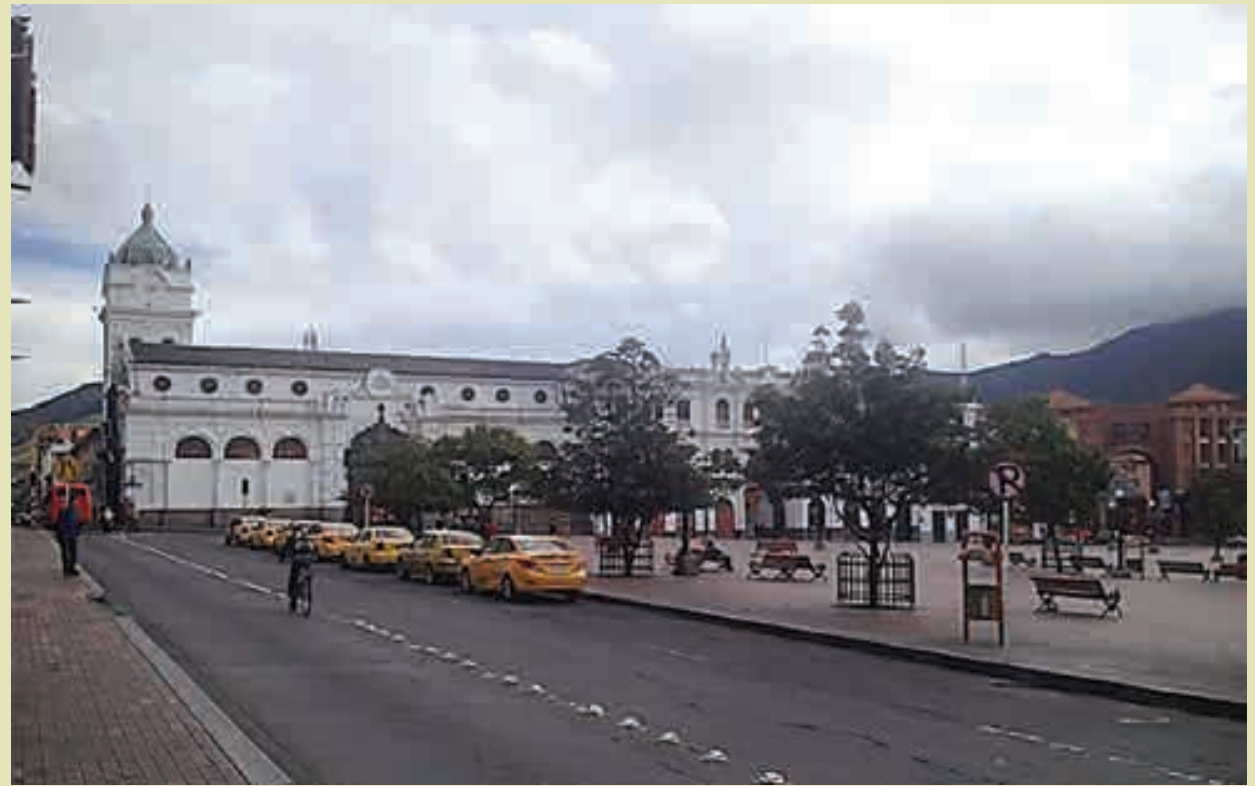

\section{RESUMEN}

La salvaguarda del patrimonio inmueble se consolida cada día más a nivel global como tema céntrico del urbanismo, debido a que cada ciudad aporta elementos históricos que en muchos casos se encuentran bajo riesgo. Tema céntrico, porque hablar de patrimonio implica enfrentarse al dilema entre “conservación y renovación". Sin duda alguna, no hay contradicción entre estas dos palabras pues bien se puede renovar una ciudad sin olvidar que las prácticas que serán asumidas en pos de su desarrollo no irán en detrimento de la conservación del patrimonio existente. Al parecer, esta directriz ha sido asumida en los procesos de planificación de las grandes ciudades de Colombia, pues tanto población como dirigentes han sabido identificar los valores presentes en los centros históricos y entenderlos a su vez como productores de bienes y servicios. Sin embargo, en ciudades intermedias como Tunja, Popayán, Pasto o Socorro aún no se consolida una clara posición frente a la salvaguarda y protección del patrimonio, ignorándose los relativos lineamientos que han sido emitidos desde hace muchas décadas en ámbito internacional, los cuales deberían hacer eco por doquier en toda Colombia. El presente artículo expone por tanto los fundamentos de esta nueva y sana tendencia por la conservación del patrimonio, tema que no debería ser asumido o entendido como complejo, sino como una práctica "lógica y natural" que implica simplemente asumir algunas medidas que integren en la esfera de la sostenibilidad las eventuales intervenciones que se pretenden proponer para renovar una ciudad y en especial aquellas zonas que han sido testigo de hechos seculares. Así bien, se inicia con un abordaje del tema internacional para proseguir con la posición de Colombia frente al tema de la conservación y protección del patrimonio y de esta manera establecer puntos de reflexión a partir de la aproximación a un estudio de caso específico: la situación del centro histórico de la ciudad de San Juan de Pasto.

\section{PALABRAS CLAVE}

Cartas de restauración, conservación, desarrollo urbano, ICOMOS, patrimonio, renovación, UNESCO. 


\section{THE DILEMMA BETWEEN CONSERVATION AND RENOVATION FOR THE URBAN DEVELOPMENT OF HISTORICAL CENTERS. AN APPROACH TO SAN JUAN DE PASTO, COLOMBIA}

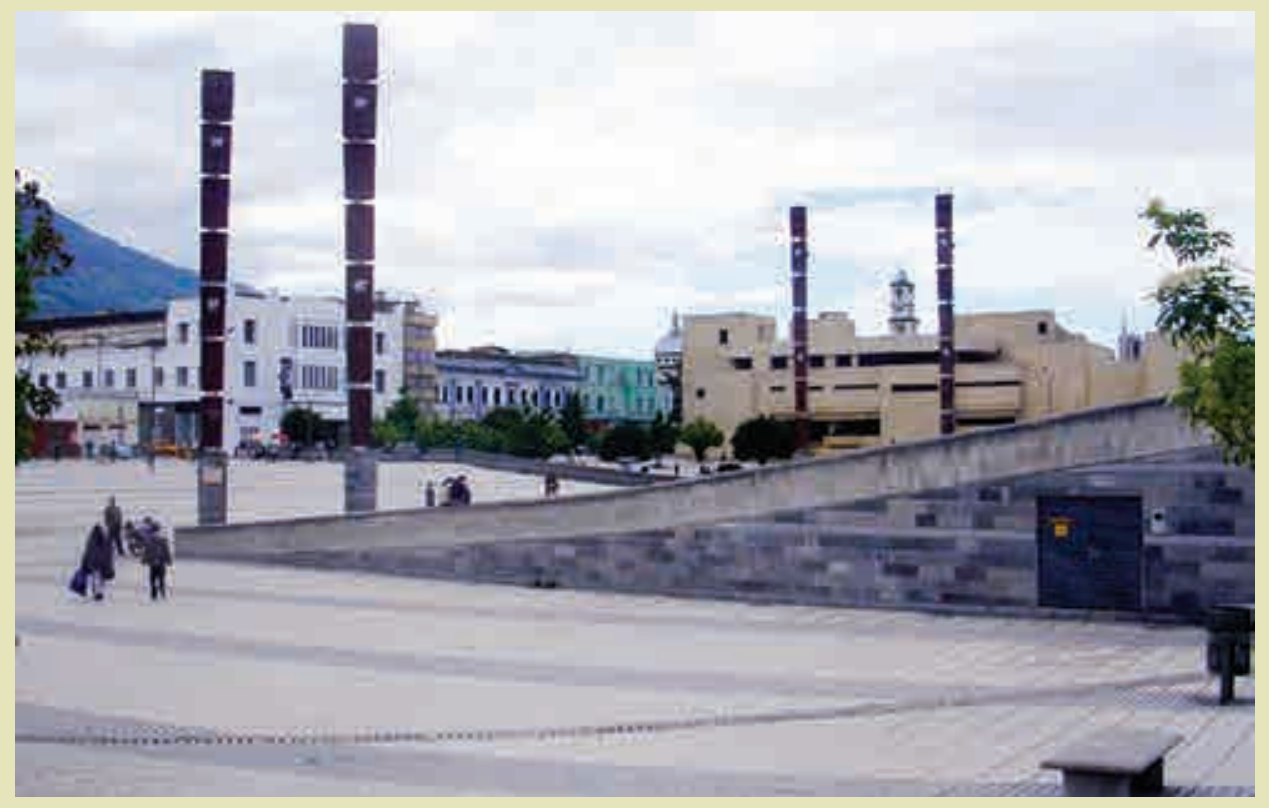

Plaza del Carnaval en San Juan de Pasto, Nariño, Colombia

Fuente: Carlos Humberto Gómez Arciniegas, 2016.

\begin{abstract}
The safeguarding of the immovable heritage is becoming more and more at a global level as a central theme in urban planning matters since each city provides historical elements that in many cases are at risk. Central theme because to speak of heritage implies facing the dilemma between "conservation and renovation". Undoubtedly, there is no contradiction between these two words, since a city can be renewed without forgetting that the practices that will be assumed in pursuit of its development will not be to the detriment of the conservation of the existing heritage. Apparently, this guideline has been assumed in the planning processes of large cities in Colombia, as both population and leaders have been able to identify the values present in historic centers and understand them, in turn, as producers of goods and services. However, in intermediate cities such as Tunja, Popayán, Pasto or Socorro a clear position has not yet been consolidated regarding the safeguarding and protection of heritage, ignoring the relative guidelines that have been issued for many decades in the international arena, which should be echo everywhere in Colombia. Therefore, this article exposes the foundations of this new and healthy trend for the conservation of heritage, a subject that should not be assumed or understood as complex but as "a logical and natural practice" which implies simply assuming some measures that integrate in the sphere of sustainability the eventual interventions that are intended to propose to renovate a city and especially those areas that have witnessed secular events. Thus, it begins with an approach to the international issue to continue with the position of Colombia in relation to the topic of conservation and protection of heritage and thus establish points of reflection from the approach to a specific case study: the situation from the historic center of the city of San Juan de Pasto.
\end{abstract}

\section{KEYWORDS}

Letters of restoration, conservation, urban development, ICOMOS, heritage, renovation, UNESCO. 


\section{INTRODUCCIÓN}

Los aspectos relativos a la conservación y la restauración del patrimonio construido están regulados internacionalmente por la Organización de las Naciones Unidas para la Educación, la Ciencia y la Cultura - UNESCO y su "brazo armado", el Consejo Internacional de Monumentos y Sitios, también conocido como ICOMOS (de la sigla en inglés International Council on Monuments and Sites), que emana periódicamente las denominadas Cartas Internacionales de la Restauración, documentos que toman un carácter formal en la Edad Contemporánea, después de la segunda década del Siglo XX (ICOMOS, 20I8). Como punto de partida en este proceso se identifica la Carta de Atenas, emanada en 1931 que será reforzada por la Carta de Venecia del 1964 y años más tarde con la Carta de Cracovia del 2000. En cualquiera de sus versiones, dichas Cartas, además de los contenidos de carácter técnico, han prestado especial atención a la preservación de la identidad de los lugares, dando importancia a muchos aspectos fundamentales desde el punto de vista socio-cultural y en especial en términos físicos de la ciudad, ya sea en sus componentes urbanos y arquitectónicos.

Sin embargo, hablar de recuperación del patrimonio construido no es prerrogativa del siglo XX, pues si bien la primera carta de restauración fue emanada en Atenas, en 1932, los registros históricos dan a conocer importantes antecedentes. Los pioneros fueron William Morris y John Ruskin, quienes en el siglo XIX empezaron a poner sobre la mesa la necesidad de preservar los monumentos arquitectónicos a través de lineamientos que guiaban posibles destrucciones o alteraciones (Choay, 1983). Lamentablemente estos postulados quedaron por mucho tiempo como voces aisladas.

Es hasta 1883 que se organiza en Roma un congreso de arquitectos e ingenieros para abordar un tema que permitiría reflexionar sobre principios para la conservación del patrimonio. El congreso originó un amplio debate entre la comunidad científica y cultural de ese gremio profesional, lo que a su vez sentó las bases para organizar otros congresos, cuyas declaratorias finales se asumieron como recomendaciones en la arena internacional (Arjones, 2015). Este breve recorrido a través de las Cartas Internacionales de la Restauración deja entrever cómo estas nacen por el deseo de reconducir la práctica de la restauración a una dimensión común en ámbito internacional, con reglas uniformes y viables, susceptibles de ser aplicadas en cualquier lugar y en cualquier tipo de intervención. Por tanto, se puede inferir que estos documentos representan los valores universales que orientan los países miembros de la UNESCO, entre ellos Colombia, a organizar sus propias leyes en el marco de esa visión común para la salvaguarda del patrimonio, aunque sin descuidar los valores intrínsecos que cada bien físico o intangible posee como parte del acervo cultural de una sociedad, elemento indivisible en la identidad de un lugar.

Este excursus histórico fundamenta el presente artículo en sus inicios para después proseguir a dejar en claro cómo la identidad del lugar está ligada al patrimonio, entendido como bien cultural que debe ser protegido y salvaguardado debido a sus múltiples valores y potenciales. Seguidamente se aborda en modo sucinto la posición que Colombia ha asumido frente al tema del patrimonio, aspecto que conducirá inexorablemente al controvertido caso del proyecto de la carrera 27 en la ciudad de San Juan de Pasto en el departamento de Nariño, confrontación que a su vez permitirá hilvanar las relativas consideraciones finales que en líneas generales abogan por una toma de conciencia y férrea responsabilidad en Colombia al momento de intervenir su patrimonio inmueble. Es este el propósito más claro del presente trabajo: resaltar el espectacular paisaje urbano de Colombia por la enorme cantidad de patrimonio histórico que allí se encuentra y, por ende, sus antiguas técnicas constructivas en tierra y guadua, su arquitectura colonial y republicana, entre otras cosas. 
Se trata más de un clamor que de un estudio técnico donde se pretenda demostrar la importancia de la conservación frente a la urgencia de la renovación para que de esta manera el lector asuma su propia posición al respecto.

\section{EL PATRIMONIO EN LA IDENTIDAD DEL LUGAR}

Se define como identidad el conjunto de rasgos propios de un individuo o de una colectividad que los caracterizan frente a los demás. Análogo a este concepto se posiciona la identidad del lugar, pues sus diferentes matices hacen referencia a una dimensión del ser humano en la que se define la identidad personal del individuo con relación al medio físico que lo circunda, a través de un complejo sistema de ideas, creencias, preferencias, sentimientos, valores y metas, combinadas tanto con las tendencias de un comportamiento, consciente e inconsciente, como con las habilidades que se forman y se reflejan en dicho entorno. En este orden de ideas, se asumen las dimensiones y las funciones espaciales como elementos concomitantes a la identidad del lugar.

Las dimensiones de ese concepto son:

- El espacio: que está vinculado al espacio físico y a la arquitectura, emocionalmente y socialmente dotado de significado.

- El tiempo: en referencia a la sedimentación de experiencias pasadas, presentes y expectativas futuras.

- Paralelamente, las funciones de ese concepto son:

- Función de reconocimiento: el componente histórico de la identidad del lugar que permite evaluar el entorno y reconocer las señales de percepción relativas a un buen nivel de seguridad y confianza. Esto permite que un previo estudio de cada lugar ayude a determinar sus niveles de seguridad.

- Función de significado: ayuda a comprender las metas, los objetivos y la funcionalidad de un determinado lugar. Junto con la función de reconocimiento, permite crear un sistema para la evaluación del contexto.

- Función de expresión: cuando el entorno físico no coincide con las expectativas de la persona interviene la función de expresión de la identidad del lugar, la cual trata de satisfacer las preferencias del habitante mediante la personalización del medio ambiente con el fin de disminuir eventuales discrepancias y las molestias allí presentes. La función de expresión es también útil para entender cómo un entorno coincide o no con la dimensión social, siendo esto la razón de las preferencias humanas, ligadas a decisiones ambientales.

Lo importante es destacar que la identidad del lugar y el vínculo de apego a los lugares están estrechamente relacionadas y esta relación se hace cada vez más fuerte en la medida que los elementos son positivos. En otras palabras, los altos niveles de apego territorial corresponden a una fuerte sintonía e identificación con el lugar. Si, por alguna razón, se dañará o modificará la identidad del lugar, no solo en términos sociales sino físicos que incluyen los componentes constructivos que conforman el paisaje arquitectónico o urbano, se disminuirá el vínculo de apego, con irreversibles consecuencias de tipo emocional y personal.

Modificando el espacio físico que propicia la identidad del lugar se corre el riesgo de dañar la parte emocional del concepto de tiempo, o sea, aquella parte que conforma emocionalmente los recuerdos estrechamente ligados a la construcción de una vida. El reto es aún más grande cuando el componente social, que se vería afectado o influenciado por las consecuencias de un proceso de cambio urbano, son niños, adolescentes o adultos jóvenes. De aquí, es posible afirmar que el verdadero patrimonio histórico "no reside" o 
no encuentra lugar en las grandes ciudades como las "megalópolis" en donde el centro histórico está inmerso en un horrible conjunto de autopistas, centros comerciales, y torres. Son, por el contrario, las ciudades de menor escala, que entre ellas mismas tienen el potencial de constituir una red patrimonial que sirve como base y sustento de la memoria histórica de una nación y por tanto de su propia y específica cultura. Alterar esa red significa menoscabar la entera memoria cultural de toda una nación.

\section{EL ENTENDIMIENTO SECULAR DEL PATRIMONIO COMO BIEN CULTURAL}

El patrimonio histórico construido pertenece al concepto de bien cultural. Sin embargo, aún existen ciertos interrogantes vigentes por disipar al momento de definir qué se entiende como bien cultural; cómo ha evolucionado el concepto desde el siglo pasado hasta hoy; cuáles son las herramientas que se poseen para lograr la protección, la recuperación o la restauración de ese valor y, naturalmente cuáles son las instituciones que trabajan al respecto a nivel internacional y local. Sin ánimo de ser exhaustivos, es posible dilucidar estos interrogantes a partir de la esencia misma del significado global que se le atribuye a los "bienes culturales": todos aquellos productos distinguidos por cada Estado como de importancia para la propia cultura, reflejados en la arquitectura, la arqueología, la literatura, el arte, la ciencia, el folklore, la etnología o la misma antropología. Se oponen a estos, por su definición, los "bienes naturales" pues estos son ofrecidos por la naturaleza, mientras que los culturales son producto del ser humano. Por consiguiente, cada país define su patrimonio cultural con el objetivo de preservar una memoria histórica, siendo esta también un concepto historiográfico afín, aunque de desarrollo relativamente reciente, cuya formulación puede atribuirse comúnmente a Pierre Nora (1984).

La memoria histórica designa el esfuerzo consciente de los grupos humanos por entroncarse con su pasado, sea este real o imaginado, valorándolo y tratándolo con especial respeto. Conocida también como memoria colectiva, está a la base de la identidad cultural de un pueblo y sirve para que en el presente y futuro no se repitan los mismos errores del pasado. En otras palabras, construye el futuro a partir de hechos acaecidos, tal como lo habían expresado pensadores, filósofos y estudios, entre estos Marco Tulio Cicerone, en De Oratore, II, 9, 36:

"Historia vero testis temporum, lux veritatis, vita memoriae, magistra vitae, nuntia vetustatis".

O sea, "la historia es el verdadero testigo de los tiempos, luz de la verdad, vida de la memoria, maestra de la vida, mensajera de la antigüedad" (Cicerón, 2013). A pesar de esta acertada definición, los historiadores concuerdan que el concepto de bien cultural y los postulados para su protección no existían antes del siglo XIX. En realidad, algunos tentativos provienen de la Edad Antigua, aunque más como una forma de aproximación inicial al tema, pero de manera muy genérica. Como ejemplos claros de esta posición es suficiente hacer referencia a las definiciones de Marco Vitruvio Polión (2008) y a sus palabras claves de la arquitectura utilitas, firmitas, venusta (fuerza, firmeza y la elegancia) o los axiomas de la época del pre-renacimiento italiano en la ciudad de Siena, en 1309, emanados en una ley denominada el Constituto del Comune di Siena, o sea, un conjunto de normas y de leyes que regulaban la vida pública, traducidas a la lengua vulgar con el fin de hacerlas compresibles e inclusivas a las personas que no conocían el latín en el municipio de Siena, actual Región Toscana (Comune di Siena, 1903). Rezaba en uno de sus apartes: 
"Chi governa deva intendere massimamente alla bellezza della città, perché la città deve essere onorevolmente dotata et guernita, tanto per cagione di diletto et allegrezza ai forestieri quanto per onore, prosperità et accrescimento de la città et de' cittadini."

De la traducción al español de este aparte en italiano antiguo, se puede apreciar cómo este documento, comparable con una constitución u otro instrumento de planificación de nuestros días, establecía que

Quien gobierna la ciudad tiene que cuidar con el máximo esfuerzo la belleza de la ciudad, porque la ciudad debe estar honorablemente equipada y adornada, ya sea por el placer y la alegría de los forasteros como por el honor, la prosperidad y el crecimiento de la ciudad y de los ciudadanos.

Desde aquel entonces, se forma en Italia una fuerte conciencia sobre la necesidad de cuidar su memoria histórica, conciencia que aún se mantiene viva y que hace de este país “la patria del concepto de conservación”.

Hoy en día, después de más de siete siglos de recorrido histórico, el concepto de "bien cultural" (material e inmaterial) y de "patrimonio histórico construido" ha cambiado mucho a causa de un incesante evolucionar. La historia de esa evolución es la misma historia de los contenidos de las cartas sobre restauración. Retomando este recorrido histórico relativo al patrimonio como bien cultural, es necesario volver a la famosa Carta de Atenas, antes citada en este artículo, la cual aparece en 1931 y pasa a la historia por ser la primera en emitirse sobre asuntos específicos de la materia (Le Corbusier y Capella, 198I). Consiste en 10 interesantes recomendaciones, las cuales merecen mencionarse para darse cuenta de su imperecedera vigencia, hecho aún más notorio si se aplican en un contexto dado del territorio nacional. Así bien, en el documento se exhorta, omitiendo las recomendaciones específicamente técnicas, a:

- Proteger al patrimonio arquitectónico.

- Equilibrar las leyes locales para que el interés privado no se sobreponga al interés público.

- Velar por la formación cultural de la juventud, impulsando la enseñanza de la historia del arte y de la arquitectura para así respetar arte y arquitectura.

Es así que se crea el primer derrotero hacia la formación de una conciencia social y la reorganización normativa con un ideal de equilibrio entre público y privado. Esta actividad internacional para la protección de monumentos y obras de arte iniciada con la Carta de Atenas en 1931 se ve interrumpida drásticamente con el estallido de la Segunda Guerra Mundial (1939-1945). La situación de desastre total después de 1945 y la consiguiente necesidad de una reconstrucción inmediata, conduce a una reevaluación de los principios de la restauración científica, de teorías cautelosas y equilibradas centradas en la intervención mínima y la adición neutral expresada por Gustavo Giovannoni y reafirmada en el documento de Atenas.

Años más tarde, se consolidaría el primer reconocimiento oficial de "patrimonio cultural" en ámbito internacional durante la Convención de La Haya, el I 4 de mayo de 1954, firmada por cuarenta Estados de todo el mundo (UNESCO, 2018). Las normas sobre el patrimonio cultural eran en su esencia disposiciones para la salvaguarda de bienes patrimoniales durante las guerras, afirmando que los atentados contra el patrimonio cultural de cada 
pueblo constituían a su vez un acto de violencia contra el patrimonio de toda la comunidad internacional. Sin embargo, estos postulados se referían a los elementos arquitectónicos, específicamente aquellos que se entendían como "monumentos".

Después de las destrucciones infligidas al patrimonio cultural (artístico, arquitectónico e histórico) de muchas naciones de Europa y el resto del mundo y la fase inmediata de la reconstrucción de posguerra, las preguntas y las adquisiciones teóricas serían objeto de una reunión promovida en 1964, en Venecia, durante el II Congreso Internacional de Arquitectos y Técnicos de Monumentos Históricos, celebrado del 25 al 3 I de mayo y en el que se formaría el ICOMOS. Sobre la base de esta profunda reflexión teórica se llega a la creación y difusión de un documento común, denominado la "Carta de Venecia", cuya definición se dio en gran parte a la contribución teórica particular de los arquitectos Roberto Pane y Piero Gazzola y el crítico de arte Cesare Brandi. El documento veneciano surge de la necesidad de renovar, profundizar y ampliar los contenidos de la anterior "Carta de Atenas" (ICOMOS, 1965).

Cabe anotar que la Carta di Venecia aún resulta muy de actualidad, aun después de la Carta análoga emitida en la 18 Asamblea General y Simposio Científico, realizado del 9 al 14 de noviembre de 2014 en Florencia, Italia, ocasión en la que se celebraron los 50 años de vigencia de la Carta de Venecia y se reafirmó la importancia y la modernidad de sus contenidos, pues las secuelas de los desastres de la Segunda Guerra Mundial, como se mencionó anteriormente, aún seguían como tema por tratar en las agendas de los estudiosos preocupados por la restauración del patrimonio histórico destruido o afectado e inclusive que por las mismas obras de reconstrucción emprendidas por los Estados vulnerados que a veces se convertían en falsos históricos (ICOMOS, 2018). Es sobre todo por esta razón que la Carta de Venecia se considera como el primer freno a esta tendencia, mediante el establecimiento de los principios de una correcta restauración, desde un punto de vista, sea filosófico o técnico. No obstante, profundizó el tema del concepto de conservación extendiéndolo al ambiente urbano, al entorno del monumento histórico.

Muchas fueron las cartas que se desarrollaron sucesivamente y todas se pueden encontrar en el sitio web de ICOMOS, entre ellas, y de particular importancia la Carta de Ámsterdam de 1975, en la que se asume la conservación del tejido territorial histórico como fundamental (ICOMOS, 20I8). Esta tendencia se reafirmaría en la Carta de Machu Picchu, documento que contribuiría además a que Europa se enterará finalmente de la importancia de América Latina, desde ese momento, se entra en un tema de absoluta vigencia: la planificación urbana. Para dar cuenta de eso, es oportuno repetir literalmente las palabras de un punto clave de esa Carta:

La planificación en el contexto contemporáneo de urbanización debe reflejar la unidad dinámica de las ciudades y sus regiones funcionales esenciales entre los barrios, distritos y otras áreas. (...) El objetivo de la planificación en general, incluyendo aspectos de índole económico, diseño, urbano y arquitectura, es finalmente la interpretación de las necesidades humanas y una eventual solución a través de un contexto de oportunidad de formas y servicios urbanos (INCP, 2017).

Por lo tanto, es más que lógico deducir que la planificación es necesaria para lograr un determinado nivel de desarrollo, siempre y cuando se haga a partir de las necesidades del pueblo y, en casos como los que se ventilan en este documento, con una visión equilibrada entre conservación y renovación. 
Los albores del siglo XXI acompañan la firma de la Carta de Cracovia en el año 2000, documento con el que de alguna manera se puede considerar completo el conjunto de las herramientas técnicas dedicadas a la conservación y a la restauración del patrimonio histórico. De hecho, se llega a considerar la aplicabilidad de los principios de conservación, no solamente a los centros históricos patrimoniales sino también al paisaje, para hablar finalmente de territorio como una totalidad que custodia los valores culturales e históricos de una sociedad. Es así que los conceptos de conservación y de restauración asumen la connotación de conceptos dinámicos que evolucionan a la par de la sociedad (INCP, 20I7).

Sobre esta línea de pensamiento, durante la Asamblea General de ICOMOS, realizada en Florencia en noviembre de 20I4, se aprobó el documento en el que se define la conservación del patrimonio histórico como un valor humano y que pone sobre la mesa un debate para reflexionar sobre algunos aspectos, de alguna manera nuevos: la relación entre conservación y turismo, la importancia de la sostenibilidad y, sobre todo, la necesidad que el proceso de conservación sea un proceso participativo desde el punto de vista social.

Los últimos avances en la materia se cristalizan en la 19 Asamblea General y Simposio Científico de ICOMOS en Nueva Delhi (India), que se llevó a cabo entre el II y I5 de diciembre de 2017. ICOMOS (2017) clasifica el evento como exitoso, con una asistencia de más de 1000 participantes. Allí se debatieron temáticas importantes relacionadas con los hechos que han venido golpeando al mundo durante los últimos años y que afectan de una u otra manera los bienes culturales, interés común que se refleja en el título del encuentro, Patrimonio y Democracia. Entre los puntos más relevantes que se trataron cabe destacar:

- La integración entre patrimonio y desarrollo urbano sostenible con la inclusión de diversas comunidades para su respectiva gestión.

- El rol del patrimonio cultural en la construcción de la paz y de la reconciliación.

- Protección e interpretación del patrimonio en la era del empoderamiento digital.

- El recorrido entre cultura-naturaleza como viaje para explorar las complejidades de las relaciones humanas en sitios naturales y culturales.

La lista podría ser aún más extensa, pero los anteriores puntos sobresalen por su importancia y actualidad, aplicables en muchos países, entre estos Colombia. Es, en síntesis, un panorama más alentador después de casi un siglo de debate, durante el cual el concepto de conservación y restauración del patrimonio histórico ha ido evolucionando desde la idea inicial de identificar el patrimonio únicamente en un singular monumento hasta una visión más amplia, o sea la del entero territorio, que incorpora en una gran esfera tópicos como la relación entre público y privado, procesos participativos y sostenibles, así como el reto de que la conservación sea una herramienta para la paz. Sin embargo, la posición de los países en la arena internacional no es del todo homogénea por lo que entender y cuantificar todo aquello que se ha logrado hasta el día de hoy podría dar pie a una extensa investigación. Hace parte de este calidoscopio de acciones la medida en que las sociedades del siglo XXI sienten la importancia $y$ asumen las recomendaciones emanadas por estudiosos, entes $y$ organizaciones internacionales.

Hasta este apartado del artículo se presenta un panorama global sobre la temática del patrimonio y cómo ha sido abordado globalmente por lo que solo queda escudriñar la aplicabilidad de lo que ya se sabe o se sabía al momento de actuar a nivel local en pos de un desarrollo sostenible de la ciudad y en este caso, la posición que se debe asumir en el eventual momento de confrontar dos aspectos que al parecer en Colombia aún están en contrapunteo, pero que bien pueden ir de la mano: conservación y renovación. 


\section{LA POSICIÓN DE COLOMBIA FRENTE AL PATRIMONIO}

Colombia pertenece al grupo de los Estados que se une a las directrices e ideales de la UNESCO e ICOMOS. De hecho, algunas ciudades, objetos arquitectónicos y eventos culturales del país se encuentran en la lista del patrimonio mundial de la UNESCO, por lo que las leyes colombianas tienen que estar conforme a las orientaciones de las cartas y declaratorias emitidas por la organización y, en su defecto, por organismos derivados, subsidiarios o aliados como ICOMOS. A partir de visitas de campo e investigaciones académicas realizadas por los autores del artículo, se pueden referir interesantes situaciones presentes en Colombia, relativas a varios casos de bienes patrimoniales históricos a diferentes escalas. Ejemplos pueden ser el Sistema Fortificado de Cartagena de Indias, el Centro Histórico de ciudades como Bucaramanga, Tunja y Manizales, pueblos o centros poblados como Barichara, Guane y Socorro e inclusive extensos territorios como el Cañón de Chicamocha (Santander), entre otros. En cualquiera de estos contextos, no es difícil identificar el interés de algunas universidades, entidades públicas y privadas o instituciones locales y gubernamentales por temas de cobertura patrimonial. Sin embargo, las investigaciones realizadas hasta el momento dejan entrever en Colombia una situación análoga a la de casi toda América Latina que puede ser explicada a partir de cuatro elementos reconocidos internacionalmente, que caracterizarían a una ciudad como verdaderamente sostenible (CEPAL, 2002):

- Sostenibilidad económica: generar ingresos y trabajo para la población.

- Sostenibilidad social: garantizar condiciones de bienestar humano (seguridad, salud, instrucción) igualmente distribuidas por clases y géneros.

- Sostenibilidad ambiental: mantener calidad y reproducibilidad de los recursos naturales.

- Sostenibilidad institucional: asegurar condiciones de estabilidad política, democracia, participación, justicia.

Ahora bien, solo en presencia de estos cuatro principios se podrá lograr la sostenibilidad de una ciudad. Sin embargo, todo parece indicar que a nivel global se trabaja relativamente poco para amalgamar el patrimonio a la dimensión social y ambiental y en grado decreciente a la escala económica, política e institucional. Concentrando la mirada en América Latina, es evidente que el continente se encuentra ad portas de este proceso y que difícilmente se pueden promediar logros satisfactorios en comparación con otros continentes, sobre todo porque el panorama se bloquea ante un horizonte incierto: sin voluntad política, no se logrará ningún tipo de sostenibilidad. Al respecto, se puede afirmar que una situación análoga tanto en Colombia como en América Latina es la inoperatividad del sistema institucional que tendría que estar al tanto de la conservación del patrimonio cultural y establecer claras prioridades y efectivas acciones de manejo.

Como ejemplo, se puede tomar el accionar del Ministerio de Cultura colombiano que a veces se sobrepone a las instituciones regionales y locales (alcaldías y departamentos) imponiendo una visión centralizada o generalizadora de las problemáticas patrimoniales, como si cualquier territorio o ciudad, grande o pequeña, compartiera muchos aspectos en común y que por tanto las intervenciones planteadas fueran adaptables a todos estos contextos. Esta apreciación se puede aclarar a partir de muchos casos en los cuales las acciones propuestas son más que discutibles. Salta a la vista, en primer lugar, el caso de Cartagena de Indias, escenario donde el Ministerio quiere implementar un Plan Especial de Manejo y Protección (PEMP) para el antiguo sistema fortificado, aislado o separado de la totalidad del casco histórico, olvidando que la declaratoria de la inclusión de Cartagena en 
la lista de ciudades patrimonio de la UNESCO habla de conjunto único. Esta divergencia ha provocado que hasta el momento Cartagena aún no cuente con un verdadero PEMP, de amplia cobertura, que regularice acciones que van en detrimento del patrimonio arquitectónico y urbanístico de la ciudad. El diagnóstico salta a la vista de cualquier desprevenido transeúnte: se permite la construcción de torres residenciales a tan solo 100 metros del Fuerte de San Fernando de Bocachica.

Situaciones discutibles también se presentan en la ciudad de Tunja, donde a pesar de propuestas adelantadas por la Alcaldía en 2013, la aplicabilidad de un PEMP no se ha dado a causa de la falta de diálogo entre esta institución y el Ministerio de Cultura, inoperancia que se traduce, por ejemplo, en la eterna discusión para lograr la peatonalización de dos calles vehiculares del núcleo histórico, como era en un principio propósito de la administración pública municipal.

También en el departamento de Santander se presentan preocupantes circunstancias, como el caso de la ciudad de Socorro, a la espera desde 2013 de un proyecto que haga frente a una latente situación de emergencia sísmica en la Basílica Menor, edificio monumental que cada día aqueja más grietas, resquebrajamientos estructurales y un pronunciado riesgo de derrumbe. En fin, la lista podría ser muy extensa por lo que la presente investigación opta por escudriñar en un caso de estudio particular que ofrece amplio margen de debate: la ciudad de San Juan de Pasto en el departamento de Nariño. Al respecto, Alberto Quintero Arturo, presidente de la Fundación Misión Obremos por Pasto, emitió una invitación de amplia cobertura para participar en el VIII Foro sobre al Centro Histórico de Pasto, dedicado a la conservación y evolución arquitectónica de esta ciudad que se constituye como un interesante estudio de caso (Diario del Sur, 2017).

\section{EL CASO DE SAN JUAN DE PASTO}

Tanto en Colombia como en América Latina, las ciudades históricas han mudado de un estilo arquitectónico colonial o republicano a lo que se podría llamar un eclecticismo historicista, eufemismo para dar a este fenómeno una definición menos peyorativa. Pasto no es ajena a esta tendencia arquitectónica que mezcla elementos de diferentes estilos de la historia del arte y de la arquitectura y que se puede materializar simplemente en un solo edificio. Varios son los casos de esta transición, la cual se pueden observar actualmente sobre la calle 18 de Pasto, entre las carreras 21 y 22, como por ejemplo, el Hotel Boutique Casa López, construcción oportunamente restaurada por la iniciativa privada (figura 3) que contrasta con el edificio situado al otro lado de la calle, el cual hoy en día aloja un conocido almacén de Pasto, por lo que su fachada ha sido alterada con vitrinas y cerramientos que cambian la estética original del edificio, dando como resultado final un edificio de "contundente estilo ecléctico" con claros falsos históricos (figura 4).

Más allá de este ejemplo puntual, el tejido urbano de Pasto ha sido escenario impotente de una constante depredación de su patrimonio cultural. Registros históricos y algunas evidencias fotográficas permiten ver con tristeza cómo reliquias históricas han desaparecido y amplias zonas con valor patrimonial han cedido el paso a una nueva identidad amparada por débiles premisas sobre el modernismo que han allanado el camino para que aparezcan en la zona histórica modernos edificios, centros comerciales, tiendas de vanguardia, y cafeterías o restaurantes con un supuesto estilo de tendencia, que a su vez ha cincelado el paisaje urbano de esta ancestral ciudad. No se trata de un eclecticismo ni de tendencias que reflejan el normal desarrollo histórico de una ciudad. Eclecticismo viene del griego eklektikós, literalmente "que elige", palabra que hace referencia a una escuela filosófica que 
Figura 3. Interior del hotel Boutique Casa López Fuente: Carlos Humberto Gómez Arciniegas y Michele Paradiso 2016

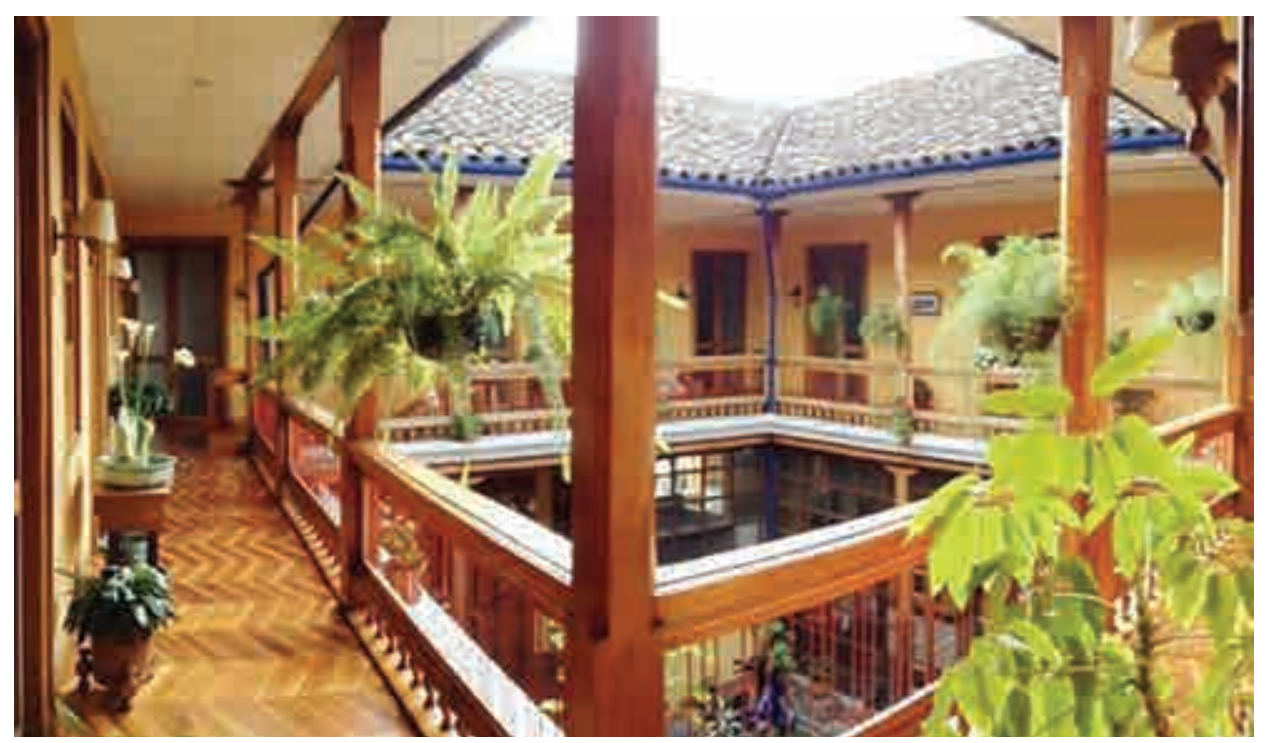

Figura 4. Edificio frente a la Casa López Fuente: Michele Paradiso, 2016

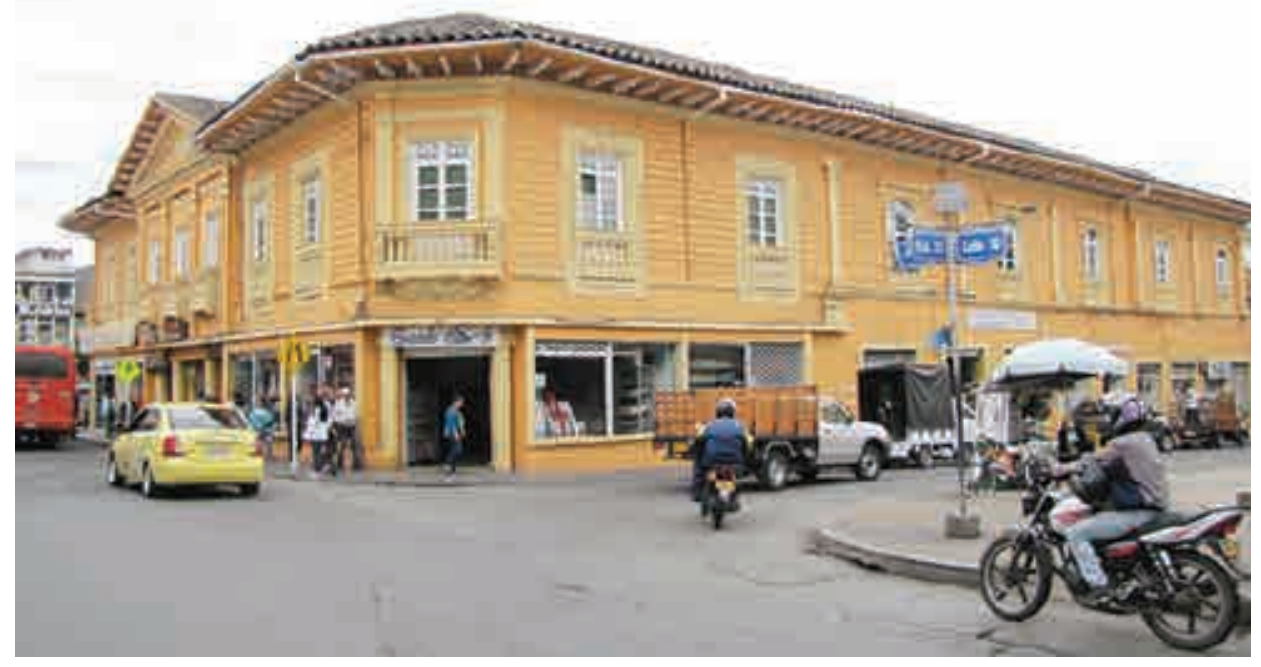

procura conciliar las doctrinas que parecen más idóneas o verosímiles, aunque procedan de sistemas diferentes. En otras palabras, un estilo fruto de una decisión, de una voluntad. Sin embargo, pareciese que en Pasto esta voluntad común no existe, ya que en su casco histórico es más que evidente un caos urbano y un popurrí de estilos que hacen perder los puntos de referencia de la memoria histórica de un pueblo y que a su vez dificulta reconocer los trazados del pasado, perdiéndose de esta manera el sentido de apego o de pertenencia a la ciudad.

De un simple recorrido por Pasto desde el punto de vista de la valorización de su patrimonio construido no es difícil individualizar antiguos edificios de rara belleza, pero en su mayoría descuidados o abandonados completamente. Iglesias, monasterios y grandes plazas parecen unirse en un solo clamor que aboga por su rescate. Pocos son los casos 
puntuales de restauración si se comparan con la gran riqueza que posee el casco antiguo de la ciudad. Sin embargo, existen ejemplos notables como el Museo Casona Taminango, complejo arquitectónico construido en el siglo XVII con sistemas típicos de esa época como lo era el de la tapia pisada y empañetados preparados a base de estiércol de res, paja picada y de una típica arcilla de la que se dice haber sido amasada por bueyes (figura 5).

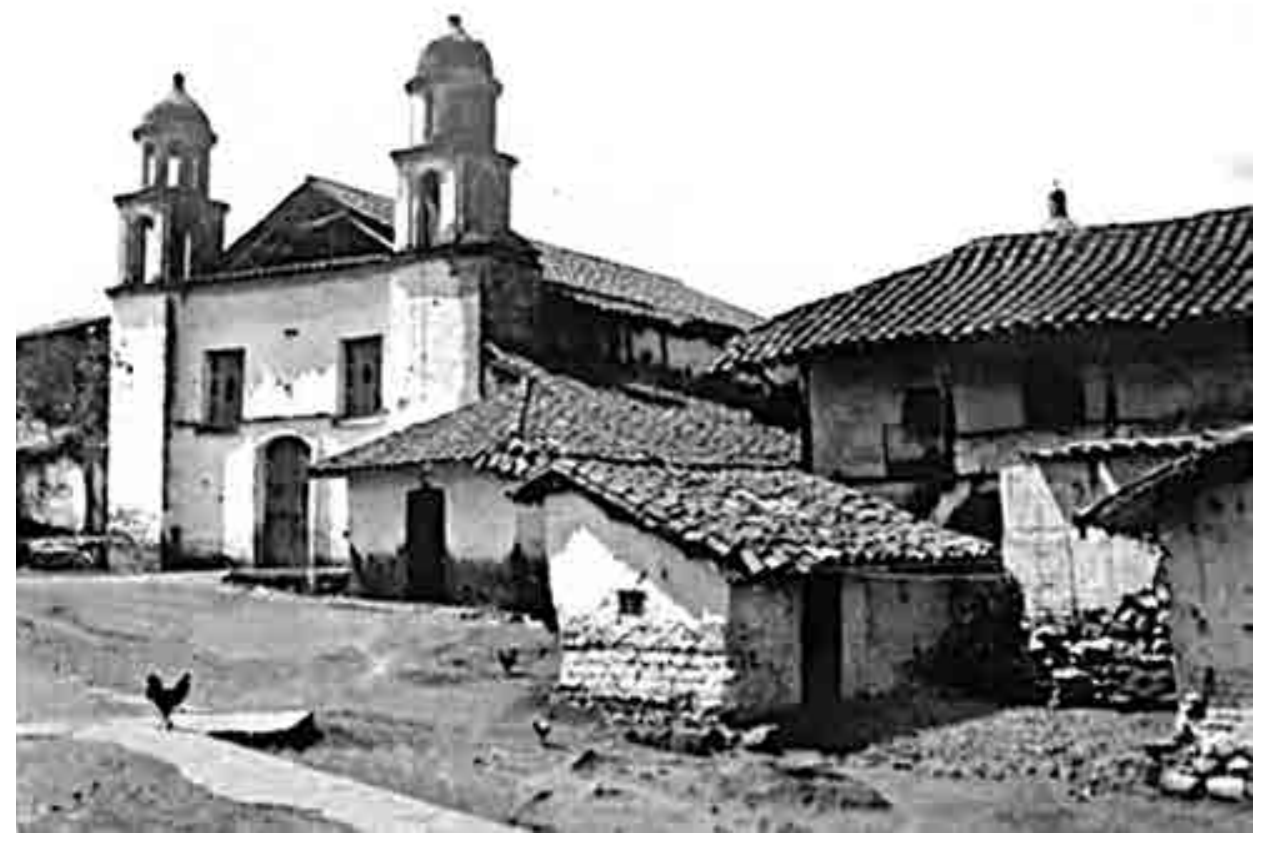

La Casona Taminango es un ejemplo de que una oportuna acción conjunta entre el sector público y privado puede actuar como mecanismo positivo para salvaguardar el patrimonio. De hecho, esta reliquia arquitectónica fue declarada Monumento Nacional a través de un específico decreto en 1971, y así conservada y restaurada por la intervención de la Fundación Museo Taminango Monasco Dachis, la cual dio el uso de Museo de Artes y Tradiciones Populares a este inmueble en julio de 1989 (figura 6).

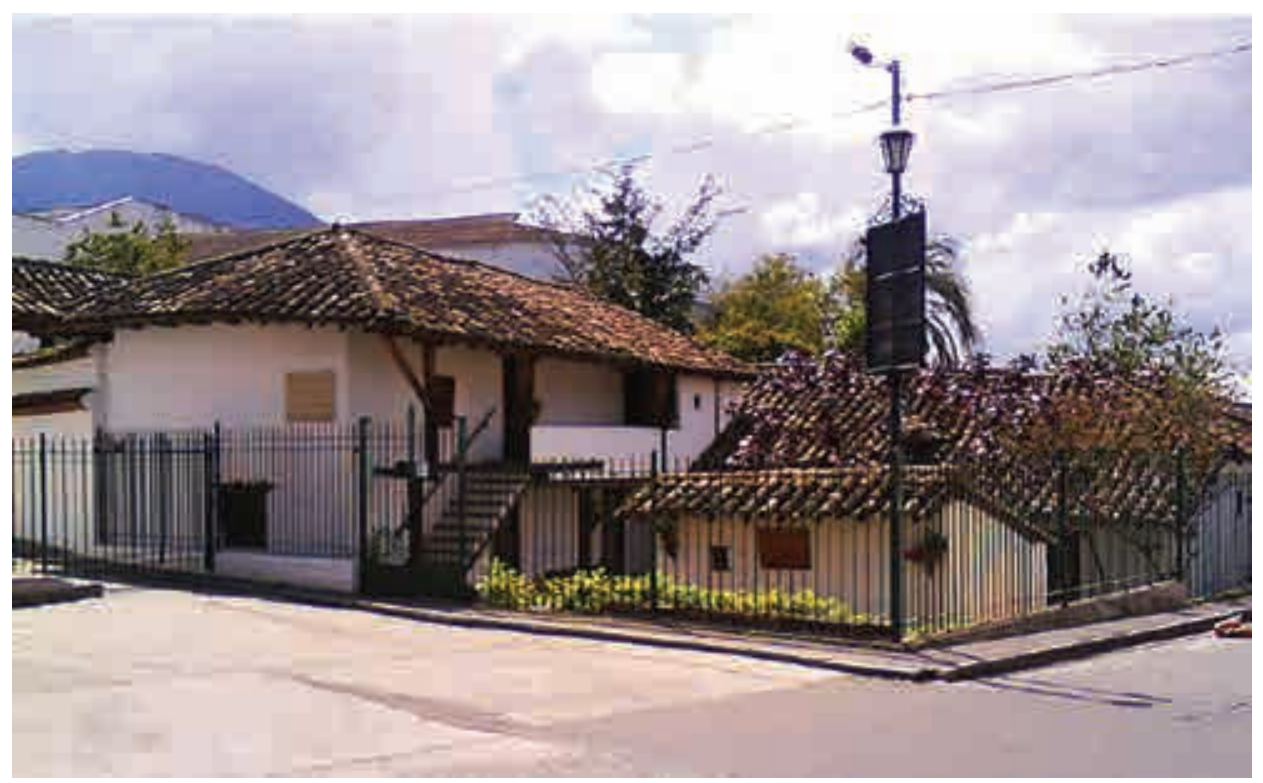

Figura 5. Estado de la Casona Taminango en 1950

Fuente: http://vidayobra.mincultura.gov.co
Figura 6. Estado actual de la Casona Taminango Fuente: Carlos Humberto Gómez Arciniegas, 2016 
También resaltan los conjuntos patrimoniales aun latentes en la ciudad como el de San Andrés, situado sobre la Carrera 27 y muchos otros en donde aún sobreviven elementos de importantísimo valor histórico, aunque esparcidas en este confuso paisaje urbano donde la actitud depredadora del urbanismo moderno amenaza contundentemente un pasado histórico rico de tradiciones. El caos vehicular, una aparente confusión social y la aparición inexplicable de edificaciones en altura, ya sea en el mismo centro o en las zonas periféricas, ocultan la belleza del marco paisajístico natural de la Cordillera de los Andes desde cualquier calle del casco histórico.

\section{El proyecto de la Carrera 27}

El afán por la modernización de la ciudad ha llevado a la administración pública a aprobar intervenciones urbanas que han dejado profundas cicatrices en el antiguo tejido urbano de la ciudad, abogando plausibles respuestas al creciente caos de la movilidad vehicular que aqueja Pasto, concomitante a la mayoría de las ciudades colombianas. Es el caso del proyecto de la Carrera 27, el cual traerá en teoría un respiro a las exigencias del flujo vehicular, pero que lamentablemente ha impactado la ciudad, no solo en términos de su patrimonio arquitectónico y estético sino también en el ámbito social. La realidad es que este nuevo eje vial se convierte en un indeseable margen urbano de la ciudad, pues corta en dos partes el casco histórico que por supuesto no termina en la Carrera 27 sino que va más allá del conjunto patrimonial de San Andrés (figura 7).

En otras palabras, una barrera que contribuye a la guetización de la ciudad y que se solapa en el pretexto de resolver un problema de transporte, calificado como prioritario pero que ignora todos los postulados de las Cartas anteriormente mencionadas, en donde se exhorta a los gobiernos a velar por una planificación integral en pos del desarrollo de la ciudad. Además, escoge una solución de estricto carácter técnico al problema, olvidando estudiar diferentes alternativas, previamente aplicadas en otros contextos internacionales, de mínimo impacto ambiental y paisajístico. Aparentemente, no se evidencia un previo proceso de participación social, juicio emitido con base en entrevistas no estructuradas realizadas a la ciudadanía residente en la zona y a transeúntes del sector. Este dictamen también se refuerza por los juicios emitidos por los medios de comunicación y algunos estudiosos que se preocupan por la salvaguardia del patrimonio. Las obras no dejan duda de este atentado al patrimonio. Las obras de ampliación del eje vial han comportado la demolición de muchas construcciones históricas, ignorando los cánones de la planificación urbana en contextos urbanos con carácter patrimonial, en el que la idea de diseño básica vela por una intervención urbana que empodere el casco histórico como corazón de la ciudad, principal depositario de la memoria histórica y la periferia, y al mismo tiempo anuncie al visitante el núcleo de la ciudad en forma liviana, avanzando suavemente desde los barrios más externos y modernos al antiguo centro, sin choques visuales o sociales. El proyecto de la Carrera 27 es sin duda un duro golpe para el patrimonio arquitectónico y urbanístico de Pasto, pero lamentablemente no hay marcha atrás. Solo queda aprender de esta triste situación para afrontar futuras ideas progresistas que van en detrimento del patrimonio histórico inmueble, valiéndose de estrategias donde se incluyan procesos de concertación y participación social antes de alterar la fisonomía de una ciudad, porque es evidente que cualquier solución que se escoja, es mejor que sea compartida con la comunidad (figura 8).

Entre tanto, se pueden reforzar acciones para aliviar las contundentes heridas infligidas a las ciudades con valor histórico, esto es situar en una posición céntrica el estudio del estilo urbano ecléctico confuso y buscar alternativas para "recoser la ciudad". 
Figura 7. Vista parcial del complejo histórico del

Templo de San Andrés

Fuente: Carlos Humberto Gómez Arciniegas,

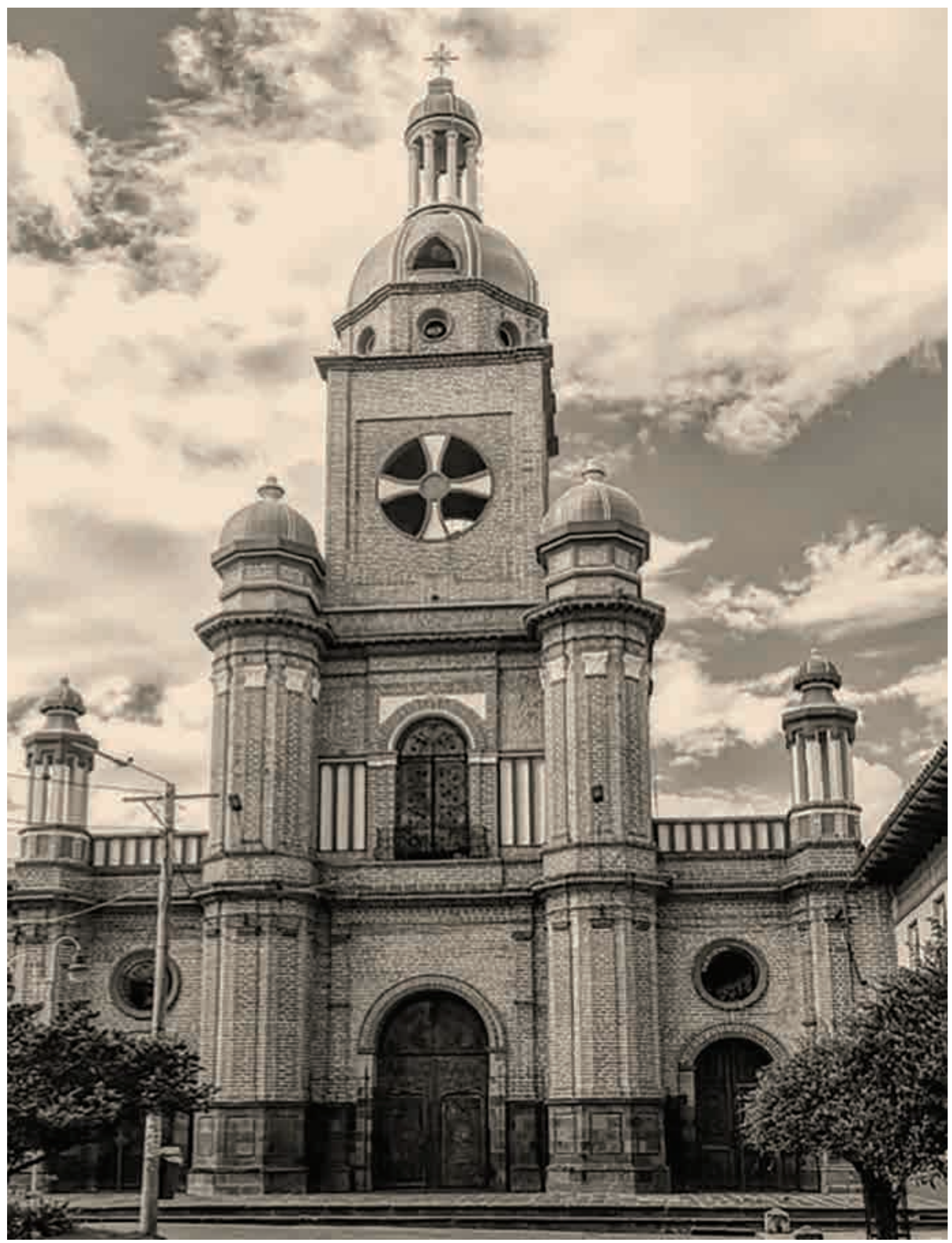

M 41 REVISTA M VOL. 14 ENERO - DICIEMBRE 2017 - FACULTAD DE ARQUITECTURA • UNIVERSIDAD SANTO TOMÁS COLOMBIA 
Figura 8. Vista aérea del proyecto de ampliación de la Carrera 27

Fuente: https://avante.gov.co/sala-de-prensa/ item/284-asi-avanzan-nuestras-obras

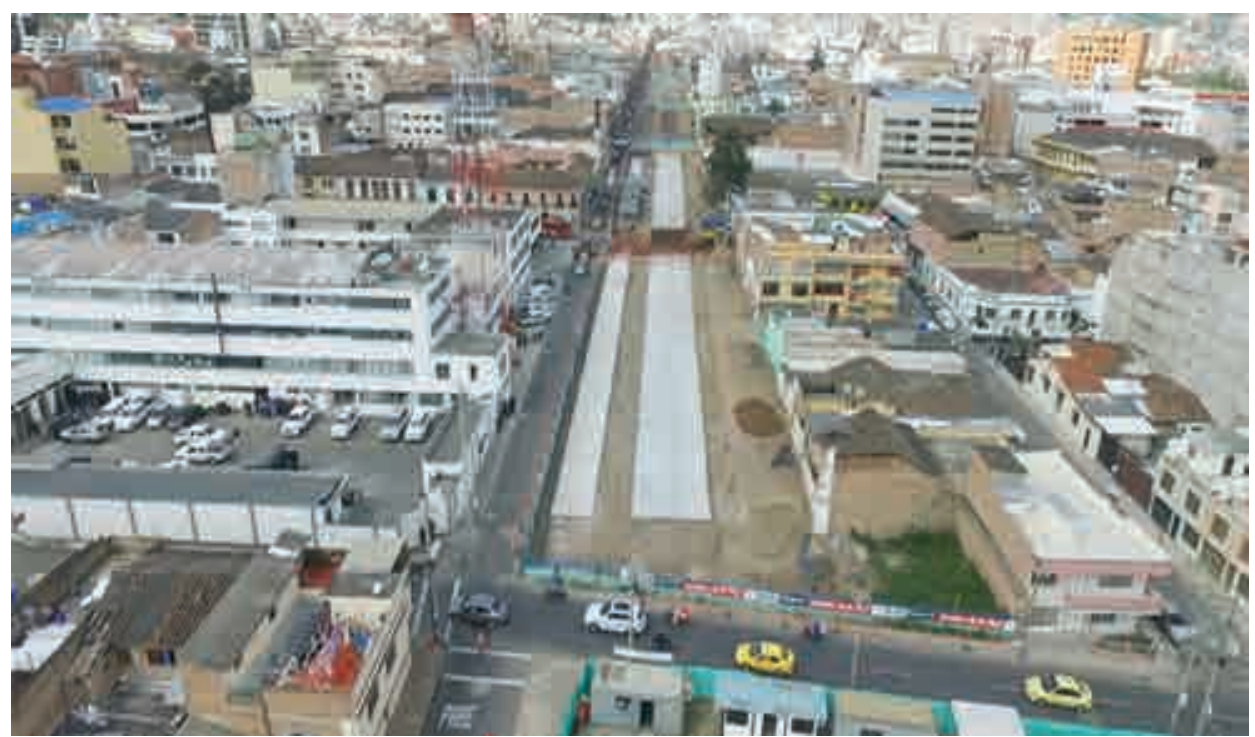

Figura 9. Panorámica parcial de la Carrera 27. A la izquierda se aprecian las acciones de demolición, infligidas al espacio privado Fuente: Michele Paradiso, 2016

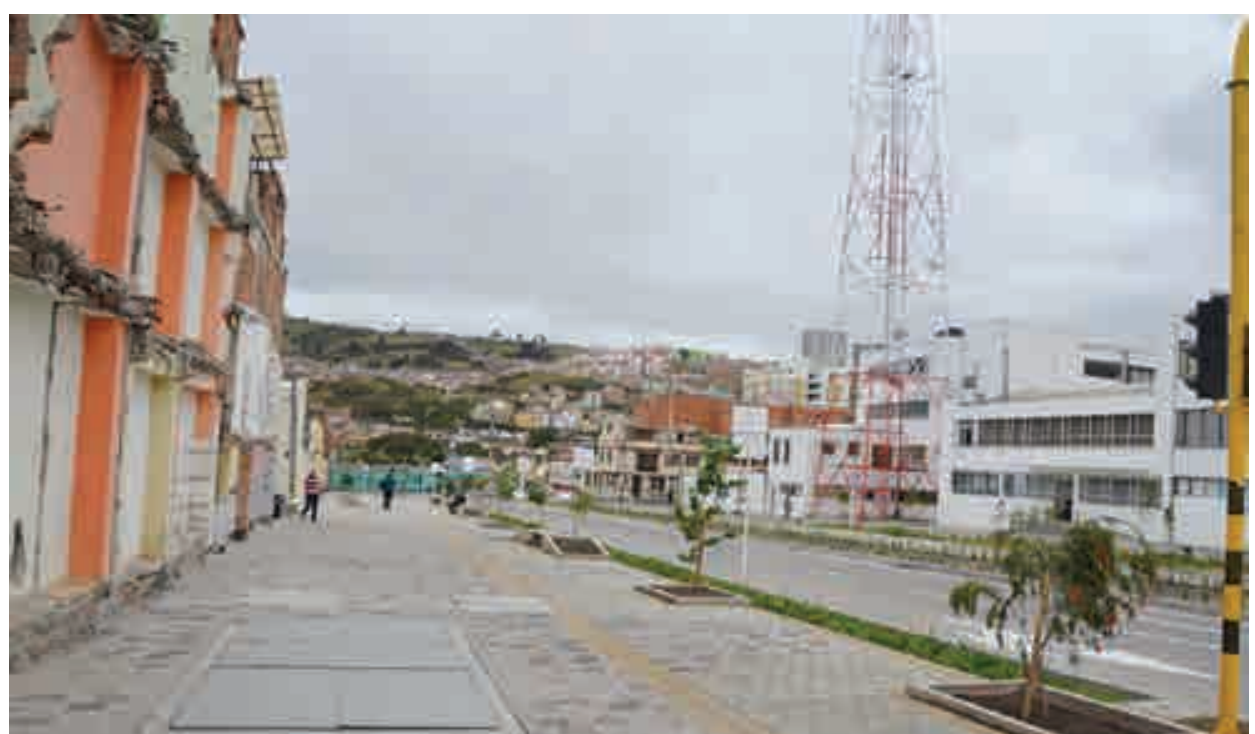

En el desastre social y urbano de las megalópolis, cualquier sociedad que viva $\circ$ trate de sobrevivir en una ciudad siempre tendrá embebido en su tejido urbano un centro fundacional y por lo tanto histórico, pues todas las ciudades lo tienen y aquellas que aún no han nacido, lo tendrán. Es tiempo de pensar y proteger aquello que aún lo permite en modo menos dispendioso como los centros poblados o las ciudades menores. Por el contrario, en aquellas ciudades de más de 20 millones de habitantes, donde prácticamente la batalla por la protección del patrimonio histórico de amplia cobertura ya se perdió, la única solución es "recoser la ciudad". Es aquí donde pequeñas ciudades como Pasto pueden convertirse en "laboratorios urbanos" destinados al rescate de los valores patrimoniales y de esta forma posicionarse como ejemplos de buenas prácticas en la cuales se utilizan medidas simples, pero de amplia aceptación social.

\section{CONCLUSIONES}

El debate sobre el contrapunteo entre conservación y renovación sigue a la orden del día en la agenda de estudiosos de la ciudad y el territorio. La visión de problemas emergentes 
en las ciudades con valores históricos, como el caso de San Juan Pasto debe ser compartida socialmente a través de comentarios y críticas constructivas que sirvan para crear momentos de reflexión y alternativas de solución. Cabe aquí mencionar iniciativas como la asociación "Obremos por Pasto", a través de las cuales se dan espacios que ayudan a identificar canales para encarrilar propuestas e inclusive "provocaciones culturales" que bien pueden llevar a consolidar la primera estrategia inicial: la reflexión para evitar decisiones apresuradas. Esto para recalcar que a veces detenerse durante una rápida competencia, que bien puede ir más allá de las propias capacidades, es mejor que continuar la carrera y perder el sentido mismo de los ideales de una sana competición. La pausa sirve para pensar en lo que se ha hecho, tanto en el plano positivo como negativo, así como retomar fuerzas y salir adelante con una visión del futuro de la ciudad, más fortalecida y sobre todo compartida.

Se trata de un futuro que debe ser pensado y planificado por la población en general, tanto por políticos como por los ciudadanos, los cuales constituyan una fuerza imperecedera que trabaje por los valores patrimoniales y que los entiendan como motor de desarrollo social y económico de la ciudad y como herencia de valor incalculable de una sociedad. No se trata de una preocupación nacida hace pocos años sino de una visión secular que data de tiempos de las civilizaciones prístinas y que va a ser retomado en consideración durante el Renacimiento y, posteriormente con la llegada de la revolución industrial y el pensamiento de preurbanistas como Pugin, Ruskin o Morris que velaban dentro de su posición culturalista por la protección del patrimonio natural y construido. Su legado será retomado por otros estudios, arquitectos y urbanistas del siglo $X X$ que a su vez sentaran las bases del accionar, sobra decir más que oportuno, de entes como la UNESCO e ICOMOS.

En relación con este último queda claro que cada día más organizaciones no gubernamentales se comprometen con la promoción de la conservación, protección, uso y mejora del patrimonio cultural mundial e ICOMOS es un ejemplo destacable. Su dedicación para el desarrollo de doctrinas comunes y la diseminación del conocimiento sin duda han contribuido a la creación de mejores técnicas para la conservación y la promoción del patrimonio cultural. Por tanto, se puede asegurar que ICOMOS ha construido un sólido marco filosófico, doctrinal y de gestión para la conservación sostenible del patrimonio en todo el mundo, el cual debe ser tenido más en cuenta en Colombia.

El caso de San Juan de Pasto, sirve para crear momentos de reflexión donde la preocupación por la conservación del patrimonio debe posicionarse en la agenda de políticos, planificadores y de la misma población. Sin embargo, esto no basta, pues queda por delante una ardua tarea relacionada con encontrar estrategias dirigidas a una concientización común que vele por hacer entender que lo antiguo no va en contra del desarrollo urbano y que este último debe ser el principal defensor del patrimonio inmueble. No es difícil encontrar buenas prácticas relacionadas con este aspecto pues toda Europa ha sabido ponerlas en marcha desde hace mucho tiempo.

Lamentablemente, el proyecto de la Carrera 27 en San Juan de Pasto depredó considerablemente buena parte de su patrimonio, tanto en su trazado urbano como en sus elementos arquitectónicos. Inútil sería escudriñar en los fundamentos que fueron asumidos por sus promotores, pero es claro que allí priman intereses económicos de varias índoles y que apoyaron lo que antes se aconsejó evitar en situaciones análogas: decisiones apresuradas. Crear un "margen urbano" de esta índole en el centro histórico de una ciudad como San Juan de Pasto no puede ser en ningún momento un hecho plausible o digno de admiración con el pretexto de un desarrollo urbano que solo piensa en la comodidad del tráfico automotor y en la especulación constructora. 
A pesar de este preocupante hecho, aún queda mucho por rescatar y proteger en esta hermosa ciudad y no solamente en hechos tangibles sino también en tradiciones y costumbres como su famoso carnaval de Blancos y Negros. Queda además la tranquilidad de saber que muchos de sus moradores se preocupan por el futuro de San Juan de Pasto, de su paisaje urbano y de sus valores patrimoniales como lo son los dueños y los trabajadores del Hotel Boutique Casa López, que en buen momento optaron por recuperar esta joya arquitectónica para sentar un precedente y un ejemplo no solo para la población de Pasto sino de otros centros intermedios de Colombia.

A la luz de lo anteriormente expuesto, se puede inferir que el camino para "recomponer" la ciudad es tomar ejemplo de otras experiencias que han sabido sortear los problemas que acarrea el desarrollo urbano y la industrialización ante la tutela del patrimonio. La experiencia europea ha permitido identificar algunas estrategias que pueden ser utilizadas para entrelazar los valores patrimoniales del centro histórico con el tejido subyacente e inclusive periférico de la ciudad y que pueden condensarse en acciones puntuales como reconstruir caminos peatonales que conecten los principales puntos de interés histórico de las ciudades; mejorar el problema de la accesibilidad a través de una red peatonal sin barreras y con un adecuado amueblamiento urbano; limitar la excesiva publicidad que la actividad comercial superpone sobre las fachadas; construir un sistema de reglas para los signos comerciales y la publicidad de las tiendas; aumentar el verde urbano y los espacios destinados al ocio y el encuentro ciudadano, como un sistema de parques y plazoletas públicas de la ciudad y regularizar los procesos de restauración de la fachada para respetar los valores originales del edifico y evitar falsos históricos.

Sin embargo, ninguna de estas estrategias pueden ser cristalizadas, si no se toma una conciencia común sobra la importancia de los centros históricos, sus grandes valores culturales $y$, sobre todo, sus infinitas potencialidades como entornos productores de bienes y servicios de gran beneficio para cualquier localidad.

\section{REFERENCIAS}

Arjones, A. (2014, decembre 3-4). Prima edizione annotata et tratotta in spagnolo dei risultati di Camilo Boito al Congresso di Roma I883. En Atti del Centenario Boitian. Camilo Boito moderno (Milano). Bergamo: Accademia di Belli Arti di Brera. (En prensa).

Choay, F. (1983). El urbanismo: Utopías y realidades. Buenos Aires: Lumen.

Cicerón, M.T. (autor) Sánchez S. E. (traductor) (20।3). El Orador. Madrid: Alianza Editorial

Comision Europea. (1994). Carta de las ciudades europeas hacia la sostenibilidad - Carta de Aalborg.

Comune di Siena. (1903). Lisini A. collaboratore, I costituto del Comune di Siena volgarizzato nel 1309-1310: edito sotto gli auspici del Ministero dell'Interno R. Archivio di Stato di Siena. Siena: Lazzeri.

Diario del Sur. (2017). Conferencistas internacionales en el VIII Foro Centro Histórico. Recuperado de http://diariodelsur.com.co/noticias/local/conferencistas-internacionales-enel-viii-foro-centro-histor-34354I.

Consejo de Europa. (1975). Declaración de Ámsterdam. Recuperado de http://www. icomoscr.org/doc/teoria/VARIOS. 1975.declaracion.amsterdam.patrimonio.arquitectonico. europeo.pdf 
ICOMOS. (1965). Carta internacional sobre la conservación y la restauración de monumentos y sitios (Carta de Venecia 1964). II Congreso Internacional de Arquitectos y Técnicos de Monumentos Históricos, Venecia 1964. Recuperado de https://www.icomos. org/charters/venice_sp.pdf

ICOMOS. (2017). Comunicado final. Resultados de la 19 Asamblea General de ICOMOS en Nueva Delhi, India, 2017. Recuperado de https://www.icomos.org/images/ DOCUMENTS/General_Assemblies/19th_Delhi_2017/19th_GA_Outcomes/GA2017_ Finalcommuniqu\%C3\%A9_FR_20I80II8.pdf

ICOMOS. (20/8). International Council on Monuments and Sites. Recuperado de https://www.icomos.org/en/

ICOMOS. (2018). I8th General Assembley and Scientific Symposium - Outcomes. Recuperado de https://www.icomos.org/en/about-icomos/governance/general-information-about-the-general-assembly/list-of-general-assemblies/ I 8th-general-assemblyflorence-2014/9-uncategorised/429- I8th-general-assembly-of-icomos-

ICOMOS Costa Rica. (2018). Declaración de Ámsterdam sobre el Patrimonio Arquitectónico Europeo.

Instituto Nacional de Cultura del Perú - INCP. (2007). Documentos fundamentales para el patrimonio cultural. Textos internacionales para la recuperación, repatriación, conservación, protección y difusión. Lima: INC.

Le Corbusier, \& Capella, J. R. (198I). Principios de urbanismo: La carta de Atenas. Chicago: Turabia.

Mincultura. (2018). Premio Nacional Vida y Obra. Pablo Morillo. Historia de una tenacidad. Recuperado de http://vidayobra.mincultura.gov.co/ediciones/2010/pablo-murillo/ vita-extensa/Paginas/default.aspx.

Naciones Unidas, Cepal. (2002). Las nuevas funciones urbanas: gestión para la ciudad sostenible. En Medio ambiente y desarrollo, No. 48. ONU, CEPAL. Santiago de Chile.

Nora, P. (1984). Le lieux de mémoire. Montevideo: Ediciones Trilce.

UNESCO. (2018). Convención para la Protección de los Bienes Culturales en caso de Conflicto Armado y Reglamento para la aplicación de la Convención 1954. Recuperado de http://portal.unesco.org/es/ev.php-URL_ID = 13637\&URL_DO=DO_TOPIC\&URL_SEC$\mathrm{TION}=20 \mathrm{I} \cdot \mathrm{html}$.

Vitruvio, M.L. (2008). Los diez libros de Arquitectura. Chicago: Turabian. 